\title{
Comparison of Metabolic Stability and Metabolite Identification of 55 ECVAM/ ICCVAM Validation Compounds between Human and Rat Liver Homogenates and Microsomes - a preliminary Analysis*
}

\author{
Olavi Pelkonen ${ }^{1}$, Ari Tolonen ${ }^{2}$, Timo Rousu ${ }^{2}$, Larissa Tursas ${ }^{1}$, Miia Turpeinen ${ }^{1}$, \\ Juho Hokkanen ${ }^{2}$, Jouko Uusitalo ${ }^{2}$, Michel Bouvier d'Yvoire ${ }^{3}$ and Sandra Coecke ${ }^{3}$ \\ ${ }^{1}$ University of Oulu Department of Pharmacology and Toxicology, Oulu, Finland; ${ }^{2}$ Novamass Ltd, Oulu, Finland; \\ ${ }^{3}$ EC Joint Research Centre ADD after EC Joint Research Centre, Institute for Health and Consumer Protection, \\ European Centre for the Validation of Alternative Methods (ECVAM), Ispra, Italy
}

\begin{abstract}
Summary
In vitro methods to produce metabolic information have increasingly been applied in toxicity risk assessment. In the current contract project of JRC/ECVAM In vitro-Toxicology Unit, 55 organic chemicals, mostly drugs and pesticides, most belonging to ECVAM/ICCVAM validation compounds, expected to be analyzable by LC-MS technique, were subjected to a feasibility study. The simple experimental setup consisted of one concentration of a chemical (25 $\mu M$ ), enzyme preparation (human or rat liver homogenate or microsomes), a set of cofactors (NADPH, UDPGA, PAPS, GSH), 4 time points $(0,15,30,60$ min, including cofactor-less tubes). Metabolites produced were analyzed and tentatively identified by LC-MS techniques.

Most of the chemicals were metabolized and metabolites were tentatively identified by TOF-MS analysis.

For some chemicals, about 10 or even more metabolites were detectable (e.g. thioridazine, verapamil, amitriptyline). Altogether 11 out of 55 did not display any metabolites under the experimental conditions of this study. Regarding the metabolites formed, there were mostly quantitative differences, but about 20 substances displayed also species-dependent qualitative differences, i.e. a major metabolite was formed in one species, but not in the other. For most chemicals, differences between microsomes and homogenates were relatively modest at least in the initial analysis.

The results demonstrate that $L C$-MS approach is feasible and rather efficient in providing useful metabolic data from a simple experimental setup. More complex analyses, e.g. quantitative assessment of differences between species or biological preparations, or in vitro-in vivo extrapolations, require more complex approaches and a collection of appropriate, preferably curated, data bases of in vivo characteristics of the studied chemicals.
\end{abstract}

Keywords: metabolic stability, metabolite formation, ECVAM/ICCVAM, validation compounds, liver preparations, human, rat, LC-MS techniques

\section{Introduction}

Elucidation of metabolic stability and metabolic routes is important in early phases of drug development, because metabolism determines to a great extent the pharmacokinetic properties of most drugs and is often behind interactions, metabolic idiosyncrasies and so on (Boobis et al 1999; Pelkonen et al 2001; Pelkonen 2002). Likewise, information on metabolic properties of any chemical is a significant piece in toxicological risk assessment. Actually in vitro methods to produce metabolic information have increasingly been applied also in chemical risk assessment (Coecke et al 2006).

Human-liver derived in vitro systems have proved useful in early studies of metabolic stability, in identifying enzymes capable of metabolising, and interacting with, drugs in use or under development and several of these systems are currently

Received $1^{\text {st }}$ April 2009; received in revised form and accepted for publication $19^{\text {th }}$ June 2009

* Project of In-Vitro Toxicology Unit/ECVAM Contract No CCR.IHCP.C432889.X 
under validation as to their predictive power to the in vivo situation (for reviews see e.g. Pelkonen et al 2009; Pelkonen et al 2002; 2005; Pelkonen and Raunio 2005).

In the current project contract with ECVAM, 55 organic chemicals, mostly drugs and pesticides, 51 of them belonging to the set of the ECVAM/ICCVAM validation compounds, was selected for in vitro metabolism studies. The goals of the investigations described in this paper were to investigate whether a relatively simple and not-too costly experimental setup were feasible and would provide appropriate data to characterise, with human and rat liver homogenate or microsomal preparations, the metabolic stability (i.e. disappearance of the parent substance from incubations with human liver preparations and appropriate cofactors for metabolism) and the appearance of metabolic products. With this approach we hope to develop, in the end, a robust and reliable testing system to obtain useful in vitro information to predict the pharmacokinetic behaviour of organic chemicals in the in vivo situation. Equally important is to assess similarities and differences between homogenate and microsomes, as well as between human and rat. The basic approach is expected to be suitable for the characterization of metabolic capability of any tissues or cell cultures from various animals or humans. Actually an analogous approach has been widely employed by us for several years in research and contract services.

\section{Materials and methods}

\section{Chemicals}

Most chemicals were supplied by ECVAM. The data required before studies were molecular weight and solubility into solvents which are compatible with the conditions in in vitro incubations with human liver preparations. Other chemicals were obtained mainly from Sigma Chemical Company (St. Louis, Missouri, USA) and Boehringer (Ingelheim, Germany) and were of the highest purity available.

\section{Human liver samples}

Human liver samples used for these studies have been obtained from kidney transplantation donors. The Ethics Committee of the Medical Faculty of the University has approved the collection of surplus human tissues for research and service purposes. Liver preparations have been extensively characterised to be used for the primary screening (sufficient model activities, no known polymorphisms, expected effects of model inhibitors). The present studies will be performed with a pool of 5 to 7 active and thoroughly characterised liver preparations.

\section{Preparation of liver homogenates and microsomes}

Normal-looking tissue was homogenised in four volumes of ice-cold phosphate buffer, $\mathrm{pH} 7.4$, and the resulting homogenate was used as such. Microsomes were separated by differential centrifugation. Briefly, homogenate was first centrifuged at $10,000 \mathrm{x} \mathrm{g}$ for $15 \mathrm{~min}$ and the supernatant was then centrifuged at $100,000 \mathrm{x} \mathrm{g}$ for $60 \mathrm{~min}$. The final microsomal pellet was suspended in $0.1 \mathrm{M}$ phosphate buffer.
Tissue samples from rats

Liver samples from Rat (Sprague-Dawley and Wistar strains, originally used for other purposes) stored in the deep freeze at $-80^{\circ} \mathrm{C}$ were used for studies. Basically the same approach was used for liver tissue samples of rat as for human, although the recovery and purity of the subcellular organelle were rigorously checked in the case of human.

\section{Incubation of a chemical}

The studied compounds were incubated with human and rat liver preparations (microsomes and homogenate) in the presence of appropriate cofactors. Asingle analytically adequate concentration $(25 \mu \mathrm{M})$ was used. In the microsome incubations, NADPH (for producing oxidized metabolites) and UDP-glucuronic acid (for producing glucuronic acid conjugates) were added, and in the homogenate incubations also GSH (for producing glutathione conjugates), PAPS (for producing sulphate conjugates) were added. Samples were taken from incubates at $0,15,30$, and $60 \mathrm{~min}$ and treated with ice-cold acetonitrile to stop the biological reactions. An incubation without cofactors was conducted in parallel and one sample was taken (60 min) as control. Additionally, for each set of study compounds, a blank incubations for each species and each preparation (cofactors but no study chemicals) were conducted. All incubations except blanks were performed in duplicate.

\section{Analysis of the samples by liquid chromatography mass spectrometry}

A Waters Alliance 2695 high performance liquid chromatographic system (Waters Corp., Milford, USA with autosampler, column oven and vacuum degasser was used with Phenomenex Luna-C18, Waters XTerra RP18, Waters XTerra MS C18, Waters XBridge Shield RP18 and Waters XBridge C18 columns (all with 2.0/2.1× $50 \mathrm{~mm}$ dimensions and 3-3.5 $\mu \mathrm{m}$ particle size). Phenomenex Luna C18 $(2 \times 4 \mathrm{~mm})$ precolumn was used with all columns. Temperature of column oven was $35^{\circ} \mathrm{C}$ and injection volumes used were 10-20 $\mu 1$. The flow was split post-column with an Acurate PostColumn Stream Splitter (LC Packings, Amsterdam, The Netherlands) with a ratio of 1:3 to MS and waste, respectively. The data was acquired with a Micromass LCT time-of-flight (TOF) mass spectrometer (Micromass Ltd., Manchester, England) equipped with a LockSpray electrospray ionization source, used both in positive and negative ion polarity. Cone voltages between $16-50 \mathrm{~V}$ were used (optimized for each compound). Capillary voltages of $3.5 \mathrm{kV}$ and $3.0 \mathrm{kV}$ were used at positive and negative ion mode, respectively. Nitrogen was used as a nebulizer and drying gas with flow rates of $100 \mathrm{l} / \mathrm{h}$ and $800 \mathrm{l} / \mathrm{h}$, respectively. The source and desolvation temperatures used were $150^{\circ} \mathrm{C}$ and $240^{\circ} \mathrm{C}$, respectively. Leucine encephalin $\left([\mathrm{M}+\mathrm{H}]^{+} \mathrm{m} / \mathrm{z}\right.$ 556.2771) and raffinose $\left([\mathrm{M}-\mathrm{H}]^{-} \mathrm{m} / \mathrm{z}\right.$ 503.1612) were used as a lock mass compounds in accurate mass measurement with positive and negative ion modes, respectively, and were delivered into LockSpray probe with syringe pump to obtain about 160 counts/s. The mass spectrometer and HPLC system were operated under Masslynx 4.0 software.

\section{Chromatographic conditions and method development}

In method development the study compounds were devided into batches of 3-10 compounds, based on their chemical struc- 
tures or acid/base properties, and LC/MS conditions for each compound were briefly optimised by acquiring test data for all compounds in a single batch at the same time. In the beginning of method development, some runs with varying gradient strength (slope of the acetonitrile or methanol proportion) were acquired, followed by runs with various aqueous phase $\mathrm{pH}$ conditions between 2.4 and 9.8 and different MS ionisation polarities to optimise the ionisation efficiency (i.e. the detection sensitivity). For compounds with poor peak shape, also the effect of different columns (see above) were tested for improving chromatographic peak shapes.

\section{Sample preparation}

The incubation samples were thawed at room temperature (RT), shaken and centrifuged for $15 \mathrm{~min}$ at $16,100 \times \mathrm{g}$ (Eppendorf 5415D, Eppendorf AG, Hamburg, Germany) and pipetted to Maximum recovery vials (Waters Corporation, Milford, Massachusetts, USA) to wait for an autosampler run.

\section{Data processing for metabolic stability and identification of metabolic transformations}

The incubations were analysed with LC/PDA/TOF-MS system as described above. For the compounds having UV activity, the PDA response in selected wavelength was used for metabolic stability analyses. For non-UV active compounds, the TOF-MS data with ${ }^{13} \mathrm{C}$ isotope ions (to avoid detector saturation) was used for metabolic stability analyses. The average of duplicate incubations was used as peak area for metabolic stability calculations.

The metabolites were mined from the acquired data by comparing the LC/TOF-MS data from metabolized sample with the negative control (blank sample), both manually and by using the MetaboLynx software. Accurate mass data was used to obtain elemental composition for all found metabolites to identify the respective biotransformations. In addition, the in-source fragment ion data obtained for some compounds were used to further elucidate the metabolite structures.

\section{Metabolite profile of a chemical}

To generate the metabolite profile for each study compound in each liver preparation, the LC/TOF-MS peak areas of each found metabolite were measured and a column chart was created for each sample. Based on the relative abundance of different metabolites and their tentative structures, proposals of biotransformation pathways were drawn, and the results of a chemical in different species and liver preparation were compared. It has to be stressed that, absolute identification and quantification was not possible, and instead, metabolite "fingerprints" were produced and compared.

\section{Results and discussion}

\subsection{Development of analytical methods}

LC/TOF-MS methods were developed with parent compounds by optimizing chromatographic behaviour (retention and peak shape) and mass spectrometric ionisation. Several reversephase HPLC columns and eluents at $\mathrm{pH}$ range 2-10 were used during these processes, and conditions giving the best performance for each substrate were used. For some of the compounds the analytical methods did not work well due to the poor retention and/or poor mass spectrometric ionisation properties of the compounds, and for these also HILIC chromatography was tested.

LC/TOF-MS methods used were well suitable for most of the 55 substances. With 4 substances, the LC/MS-MS methods had to be used due to poor specificity or sensitivity of the LC/TOFMS approach. Suitability of LC/MS for most analyses was naturally expected, because the pre-selection of compounds from the list of all ECVAM/ICCVAM validation compounds was mostly done on the basis of analytical feasibility. The most notable exceptions were compounds with a large number of halogen substituents, relatively small molecules and those substances with weak ionization properties.

A detailed and comprehensive description of analytical methods for each studied substance is under preparation and will be published in due course.

\subsection{Comprehensive categorical survey of results}

We performed an initial, principally categorical survey and comparison of all the results obtained. These assessments are collected in Table 1.

Tab. 1: Comprehensive categorical survey of the results

\begin{tabular}{|c|c|c|c|c|c|}
\hline & & \multicolumn{2}{|c|}{ Apparent disappearance $^{1}$} & \multicolumn{2}{|c|}{ Metabolites formed $^{2}$} \\
\hline Compound & Preparation & Human & Rat & Human & Rat \\
\hline \multirow{2}{*}{ Carbamazepine } & Homogenate & very slow & slow & 1 & 1 \\
\hline & Microsome & very slow & very slow & 1 & 1 \\
\hline \multirow{2}{*}{ Amitriptyline } & Homogenate & very slow & very fast & $4(1)$ & $9(4)$ \\
\hline & Microsome & very slow & very fast & $4(1)$ & $9(5)$ \\
\hline \multirow{2}{*}{ Digoxin } & Homogenate & very slow & very slow & none & none \\
\hline & Microsome & very slow & very slow & none & none \\
\hline \multirow{2}{*}{ Orphenadrine } & Homogenate & slow & fast & 3 & $4(1)$ \\
\hline & Microsome & slow & moderate & 3 & $4(1)$ \\
\hline \multirow{2}{*}{ Propranolol } & Homogenate & moderate & fast & $5(1)$ & $7(3)$ \\
\hline & Microsome & slower & slow & $5(1)$ & $7(3)$ \\
\hline
\end{tabular}


Tab. 1: Comprehensive categorical survey of the results (continued)

\begin{tabular}{|c|c|c|c|c|c|}
\hline \multirow[b]{2}{*}{ Compound } & \multirow[b]{2}{*}{ Preparation } & \multicolumn{2}{|c|}{ Apparent disappearance $^{1}$} & \multicolumn{2}{|c|}{ Metabolites formed $^{2}$} \\
\hline & & Human & Rat & Human & Rat \\
\hline \multirow{2}{*}{ Methadone } & Homogenate & slow & moderate & $1(1)$ & $1(1)$ \\
\hline & Microsome & slow & moderate & $1(1)$ & $1(1)$ \\
\hline \multirow{2}{*}{ Thioridazine } & Homogenate & moderate & moderate & $11(1)$ & $17(3)$ \\
\hline & Microsome & moderate & moderate & $11(1)$ & $17(3)$ \\
\hline \multirow{2}{*}{ Maprotiline } & Homogenate & slow & moderate & 1 & $4(1)$ \\
\hline & Microsome & slow & slow & 4 & $9(3)$ \\
\hline \multirow{2}{*}{ Diphenhydramine } & Homogenate & slow & moderate & 3 & $6(1)$ \\
\hline & Microsome & slow & moderate & 3 & $6(2)$ \\
\hline \multirow{2}{*}{ Haloperidol } & Homogenate & slow & slow & $6(2)$ & $6(2)$ \\
\hline & Microsome & slow & slow & $6(2)$ & $7(3)$ \\
\hline \multirow{2}{*}{ Atropine } & Homogenate & very slow & fast & 3 & $5(1)$ \\
\hline & Microsome & very slow & slow & 2 & $5(1)$ \\
\hline \multirow{2}{*}{ Disopyramide } & Homogenate & slow & slow & 1 & 3 \\
\hline & Microsome & very slow & slow & 1 & 3 \\
\hline \multirow{2}{*}{ Diphenylhydantoin } & Homogenate & very slow & slow & 1 & 1 \\
\hline & Microsome & very slow & slow & 1 & 1 \\
\hline \multirow{2}{*}{ Warfarin } & Homogenate & slow & slow & 3 & 4 \\
\hline & Microsome & slow & slow & 3 & 3 \\
\hline \multirow{2}{*}{ Chloramphenicol } & Homogenate & $?$ & moderate & none & 1 \\
\hline & Microsome & $?$ & very slow & none & 1 \\
\hline \multirow{2}{*}{ Rotenone } & Homogenate & moderate & fast & 7 & 7 \\
\hline & Microsome & moderate & moderate & 8 & 8 \\
\hline \multirow{2}{*}{ Diethylphalate } & Homogenate & very fast & very fast & 1 & 1 \\
\hline & Microsome & very fast & very fast & 1 & 1 \\
\hline \multirow{2}{*}{ Dibutylphtalate } & Homogenate & very fast & very fast & $2(1)$ & $2(1)$ \\
\hline & Microsome & very fast & very fast & $2(1)$ & $3(1)$ \\
\hline \multirow{2}{*}{ Ibuprofen } & Homogenate & fast & moderate & 1 & 1 \\
\hline & Microsome & moderate & moderate & 1 & 1 \\
\hline \multirow{2}{*}{ Gibberellic acid } & Homogenate & no met & very slow & none & none \\
\hline & Microsome & no met & no met & none & none \\
\hline & Homogenate & very fast & very fast & $2(1)$ & $2(1)$ \\
\hline Propylparaben & Microsome & very fast & very fast & $3(1)$ & $3(1)$ \\
\hline Nirotine & Homogenate & moderate & moderate & $3(1)$ & 1 \\
\hline Nicotıne & Microsome & slow & slow & $3(1)$ & none \\
\hline & Homogenate & moderate & moderate & $5(1)$ & $6(1)$ \\
\hline Quinıdine & Microsome & slow & slow & $5(1)$ & $5(1)$ \\
\hline & Homogenate & fast & fast & $8(2)$ & $9(2)$ \\
\hline Verapamil & Microsome & moderate & moderate & $7(2)$ & $8(2)$ \\
\hline Піэгором & Homogenate & slow & moderate & $3(2)$ & $7(2)$ \\
\hline Diazepam & Microsome & slow & moderate & $3(2)$ & $6(3)$ \\
\hline Molathion & Homogenate & rapid & rapid & 4 & 4 \\
\hline Malathion & Microsome & fast & fast & 4 & 3 \\
\hline Phonghorbitol & Homogenate & none & slow & none & none \\
\hline Phenobarbital & Microsome & none & slow & none & none \\
\hline Dontrharbito & Homogenate & very slow & slow & none & 2 \\
\hline Pentobarbital & Microsome & very slow & slow & none & 1 \\
\hline & Homogenate & slow & moderate & 3 & 5 \\
\hline Fenpropathrin & Microsome & moderate & moderate & 4 & 5 \\
\hline & Homogenate & moderate & moderate & $6(3)$ & 3 \\
\hline Chlorpyritos & Microsome & moderate & moderate & $2(1)$ & $2(1)$ \\
\hline & Homogenate & moderate & moderate & $3(1)$ & 2 \\
\hline Carbaryl & Microsome & slow & slow & $4(2)$ & 4 \\
\hline
\end{tabular}


Tab. 1: Comprehensive categorical survey of the results (continued)

\begin{tabular}{|c|c|c|c|c|c|}
\hline \multirow{2}{*}{ Compound } & \multirow[b]{2}{*}{ Preparation } & \multicolumn{2}{|c|}{ Apparent disappearance $^{1}$} & \multicolumn{2}{|c|}{ Metabolites formed $^{2}$} \\
\hline & & Human & Rat & Human & Rat \\
\hline \multirow{2}{*}{ Cycloheximide } & Homogenate & slow & slow & 1 & none \\
\hline & Microsome & very slow & very slow & 1 & none \\
\hline \multirow{2}{*}{ Nalidixic acid } & Homogenate & slow & slow & $2(1)$ & 1 \\
\hline & Microsome & slow & very slow & $2(1)$ & 1 \\
\hline \multirow{2}{*}{ Antipyrine } & Homogenate & slow & slow & none & none \\
\hline & Microsome & very slow & slow & none & none \\
\hline \multirow{2}{*}{ Caffeine } & Homogenate & very slow & very slow & 1 & none \\
\hline & Microsome & very slow & very slow & none & none \\
\hline \multirow{2}{*}{ Theophylline } & Homogenate & very slow & \multicolumn{2}{|c|}{ very slow none none } & \\
\hline & Microsome & very slow & very slow & none & none \\
\hline \multirow{2}{*}{ Procainamide } & Homogenate & very slow & moderate & 1 & $3(1)$ \\
\hline & Microsome & very slow & slow & none & $3(2)$ \\
\hline \multirow{2}{*}{ Isoniazide } & Homogenate & none & none & none & none \\
\hline & Microsome & none & none & none & none \\
\hline \multirow{2}{*}{ Aminopterin } & Homogenate & none & none & none & none \\
\hline & Microsome & none & none & none & none \\
\hline \multirow[b]{2}{*}{ Aflatoxin B1 } & Homogenate & moderate & slow & $3(1)$ & $2(1)$ \\
\hline & Microsome & moderate & slow & $2(1)$ & $2(1)$ \\
\hline \multirow{2}{*}{ Colchicine } & Homogenate & slow & slow & $2(1)$ & $3(1)$ \\
\hline & Microsome & slow & slow & $2(1)$ & $3(1)$ \\
\hline \multirow{2}{*}{ Strycknine } & Homogenate & moderate & moderate & 4 & 6 \\
\hline & Microsome & slow & slow & 4 & 4 \\
\hline \multirow{2}{*}{ Physostigmine } & Homogenate & slow & fast & $5(1)$ & $7(3)$ \\
\hline & Microsome & slow & moderate & $3(1)$ & $5(2)$ \\
\hline \multirow{2}{*}{ Fipronil } & Homogenate & moderate & slow & 1 & 1 \\
\hline & Microsome & slow & slow & 1 & 1 \\
\hline \multirow{2}{*}{ Dextropropoxifene } & Homogenate & moderate & very fast & $4(1)$ & $11(1)$ \\
\hline & Microsome & moderate & fast & $4(1)$ & $11(1)$ \\
\hline \multirow[b]{2}{*}{ Methylphenidate } & Homogenate & slow & slow & $2(1)$ & $4(1)$ \\
\hline & Microsome & slow & slow & 1 & $3(1)$ \\
\hline \multirow{2}{*}{ Aminoglutethimide } & Homogenate & slow & moderate & 1 & none \\
\hline & Microsome & slow & moderate & none & 1 \\
\hline Diuron & Homogenate & moderate & slow & $2(1)$ & 1 \\
\hline Diuron & Microsome & slow & slow & $3(1)$ & 1 \\
\hline & Homogenate & moderate & fast & $7(2)$ & $7(2)$ \\
\hline Metalaxyl & Microsome & slow & moderate & $3(2)$ & $4(1)$ \\
\hline & Homogenate & slow? & slow? & 1 & 2 \\
\hline Fenvalerate & Microsome & slow? & slow? & 2 & 4 \\
\hline & Homogenate & fast & $?$ & 5 & 3 \\
\hline Abamectin & Microsome & moderate & moderate & 6 & 3 \\
\hline & Homogenate & very slow & very slow & none & none \\
\hline Amphetamine & Microsome & very slow & very slow & none & none \\
\hline & Homogenate & very slow & very slow & none & none \\
\hline Busulfan & Microsome & very slow & very slow & none & none \\
\hline & Homogenate & very slow & very slow & 1 & 1 \\
\hline Paracetamol & Microsome & very slow & very slow & none & none \\
\hline & Homogenate & moderate & fast & 1 & 1 \\
\hline Parathion & Microsome & moderate & slow & 1 & 1 \\
\hline
\end{tabular}

${ }^{1}$ Categories for substrate depletion: very slow (<5\%), slow (5-19\%), moderate $(20-50 \%)$, fast $(50-80 \%)$, very fast $(>80 \%)$ in the first 15 -min incubation. Consistency of substrate loss curve was generally assessed on the basis of the whole substrate depletion curve over 60 minutes.

${ }^{2}$ The first figure is the number of all identified metabolites; the figure in parentheses means the number of major metabolites. 
Categories for substrate depletion were the following: very slow $(<5 \%)$, slow $(5-19 \%)$, moderate $(20-50 \%)$, fast $(50-80 \%)$, very fast $(>80 \%)$ in the first 15 -min incubation. Substrate loss was generally estimated on the basis of the whole substrate depletion curve over 60 minutes. Sometimes time-dependent changes were not consistent and a certain degree of uncertainty remains for some compounds.

Metabolites are classified into major and minor metabolites on the basis of relative peak areas. It is of importance to stress that signal strengths may be different for various types of metabolites and consequently, assignments into major and minor metabolites are only provisional.

\subsection{Substrate depletion}

Metabolic stability was very variable; for a number of substrates, loss was negligible over the incubation time (e.g. digoxin, gibberellic acid), for some others, no substrate was detectable after 15 minutes (e.g. diethyl and dibutyl phthalate, propylparaben). It was possible to calculate an apparent intrinsic clearance for most of the chemicals, although for those at extreme ends (i.e. very stable or labile chemicals) only "limit" clearances (smaller or larger than a certain value) were possible to estimate. In Table 2 we have collected the chemicals which displayed either very slow $(<5 \%$ depleted) or very fast $(>95 \%)$ depletion in incubations. However, a more quantitative approach necessitates also a value for the binding of each substance with microsomes or homogenates, to be used for the calculation of unbound intrinsic clearances. Because of large differences in lipid solubility and ionization and other properties of the chemicals studied, measurement of microsomal or homogenate binding for some substances proved very difficult and further investigations have to be performed. It has to be stressed that some substances may bind also to plastic or glass and give erroneous values for the unbound fraction.

\section{Differences between human and rat}

With respect to metabolic stability, most chemicals displayed relatively similar behaviour in human and rat preparations, i.e.

Tab. 2: Very slowly ( $<5 \%$ in 15 minutes, i.e. difficult to reliably study) or very quickly ( $>95 \%$ in 15 minutes) depleted chemicals in human and rat liver homogenates and microsomes

\begin{tabular}{|c|c|}
\hline Chemicals & Remarks \\
\hline \multicolumn{2}{|c|}{ Very Slowly Depleted Compounds } \\
\hline \multicolumn{2}{|c|}{ Human liver homogenates (h)/microsomes (m) } \\
\hline Carbamazepine & Also rat $\mathrm{m}$ \\
\hline \multicolumn{2}{|l|}{ Amitriptyline } \\
\hline Digoxin & Also rat \\
\hline \multicolumn{2}{|l|}{ Atropine } \\
\hline \multicolumn{2}{|l|}{ disopyramide $\mathrm{m}$} \\
\hline \multicolumn{2}{|l|}{ Diphenylhydantoin } \\
\hline \multicolumn{2}{|l|}{ Gibberellic acid } \\
\hline \multicolumn{2}{|l|}{ Phenobarbital } \\
\hline \multicolumn{2}{|l|}{ Pentobarbital } \\
\hline Cycloheximide $\mathrm{m}$ & Also rat $\mathrm{m}$ \\
\hline \multicolumn{2}{|l|}{ Antipyrine $\mathrm{m}$} \\
\hline Caffeine & Also rat \\
\hline Theophylline & Also rat \\
\hline Aminopterin & Also rat \\
\hline Amphetamine & Also rat \\
\hline Busulfan & Also rat \\
\hline Paracetamol & Also rat \\
\hline \multicolumn{2}{|c|}{ Rat liver homogenates/microsomes (not including above mentioned) } \\
\hline \multicolumn{2}{|l|}{ Chloramphenicol m } \\
\hline \multicolumn{2}{|l|}{ Nalidixic acid $\mathrm{m}$} \\
\hline \multicolumn{2}{|c|}{ Quickly depleted chemicals } \\
\hline \multicolumn{2}{|c|}{ Human liver homogenates/microsomes } \\
\hline Diethyl phthalate & Also rat \\
\hline Dibutyl phthalate & Also rat \\
\hline Propylparaben & Also rat \\
\hline \multicolumn{2}{|c|}{ Rat liver homogenates/microsomes } \\
\hline \multicolumn{2}{|l|}{ Amitriptyline } \\
\hline Dextropropoxiphene $\mathrm{h}$ & \\
\hline
\end{tabular}


differences were either negligible or from minor to moderate at the most. However, there were several notable exceptions, the most conspicuous ones e.g. amitriptyline, aflatoxin B1, dextropropoxifene (in homogenates), and parathion (in homogenates).

\section{Differences between microsomes and homogenates}

For most chemicals, differences between microsomes and homogenates in metabolic stability were relatively modest at least in the initial analysis. Exceptions included propranolol, nicotine, quinidine, verapamil, carbaryl (quite large quantitative differences), chloramphenicol, and parathion (only in rat). However, it has to be stressed that on-going calculations of intrinsic clearances employing scaling factors and unbound substrate concentrations may change the initial results at least to a certain extent.

\subsection{Metabolite formation}

Most of the chemicals were metabolized to one or more metabolites, which were tentatively identified on the basis of the exact mass (Table 1). Because of curation of this huge data base, identifications will be published later and compared with published findings of in vitro and in metabolism. For some drugs, close to 10 or even more metabolites were detectable (e.g. thioridazine, verapamil, amitriptyline).

Some chemicals, such as caffeine and theophylline displayed unexpectedly very little metabolism. A potential reason might have been that a low substrate concentration was used. Caffeine and theophylline are known to be low-affinity substrates for their metabolizing enzymes. Another interesting chemical was paracetamol, for which only one metabolite, a sulphate conjugate, was detectable in homogenates, but (expectedly) not in microsomes. Again, a possible reason was a low substrate concentration used.

We have collected the compounds with no detectable metabolites or a metabolite detectable in 1 or 2 preparations in Table 3.
Altogether 11 out of 55 compounds did not show any metabolites. Some of these compounds may be genuinely "non-metabolizable", but some others, e.g. antipyrine, caffeine, theophylline, amphetamine and paracetamol are extensively metabolized by the hepatic enzymes. As said above, it is probable that in these cases the reason for the lack of metabolites is due to the low substrate concentration, resulting in a very low production of a metabolite(s) in relations to the sensitivity of the assay.

\section{Differences between human and rat}

We have compiled in Table 4 a summary of differences in the presence/absence of metabolites and major/minor metabolites between human and rat, on one hand, between homogenates and microsomes, on the other.

Regarding the metabolites formed, there were mostly quantitative differences, but several compounds displayed also speciesdependent qualitative differences, i.e. a metabolite was detectable in one species, but not in the other. Especially noteworthy was that about 20 compounds, i.e. about $30 \%$ of all the substances, displayed a species difference in the formation of major metabolite(s) and in about half of the compounds at least some minor metabolites were different between humans and rats. However, these qualitative differences may also have been a consequence of the sensitivity of the analytical method, and it is possible that different metabolite profiles may have been detected with a more sensitive method. For example, dextropropoxiphene displayed quite striking both quantitative and qualitative differences between human and rat preparations.

\section{Differences between microsomes and homogenates}

For most chemicals, differences between microsomes and homogenates were relatively modest at least in the initial analysis. Exceptions included paracetamol (sulphate conjugation is not a microsomal reaction) and metalaxyl (more metabolites pro-

Tab. 3: Chemicals with no detectable metabolites or a metabolite detectable in only 1 or 2 preparations in human and rat liver homogenates and microsomes

\begin{tabular}{|l|l|}
\hline Chemicals & Remarks \\
\hline No detectable metabolites & \multicolumn{2}{l|}{} \\
\hline Human liver homogenates and microsomes & Also rat \\
\hline Digoxin & Also rat \\
\hline Gibberellic acid & Also rat \\
\hline Phenobarbital & \\
\hline Pentobarbital & Also rat \\
\hline Antipyrine & 1 metabolite only in human homogenate \\
\hline Caffeine & Also rat \\
\hline Theophylline & Also rat \\
\hline Aminopterin & Also rat \\
\hline Amphetamine & Also rat \\
\hline Busulfan & 1 metabolite detectable only in human and rat homogenate \\
\hline Paracetamol & \\
\hline Rat liver homogenates and microsomes (not including above mentioned) & \\
\hline Pentobarbital & \\
\hline Cycloheximide & \\
\hline
\end{tabular}


Tab. 4: Similarities and differences in the presence/absence and major/minor metabolite(s) between human and rat liver homogenates and microsomes

\begin{tabular}{|l|c|c|c|c|}
\hline & $\begin{array}{l}\text { Human homogenate } \\
\text { vs microsomes }\end{array}$ & $\begin{array}{l}\text { Rat homogenate vs } \\
\text { microsomes }\end{array}$ & $\begin{array}{l}\text { Homogenate } \\
\text { human vs rat }\end{array}$ & $\begin{array}{l}\text { Microsomes } \\
\text { human vs rat }\end{array}$ \\
\hline $\begin{array}{l}\text { No metabolites } \\
\text { detectable }\end{array}$ & 10 & 10 & 8 & 10 \\
\hline $\begin{array}{l}\text { metabolite(s) in one, } \\
\text { but not in the other }\end{array}$ & 5 & 3 & 6 & 6 \\
\hline only one metabolite & 8 & 9 & 14 & 14 \\
\hline $\begin{array}{l}\text { major metabolite(s) } \\
\text { same }\end{array}$ & 21 & 18 & 20 & 17 \\
\hline $\begin{array}{l}\text { major metabolite(s) } \\
\text { different }\end{array}$ & 10 & 15 & 2 & 2 \\
\hline $\begin{array}{l}\text { minor metabolite(s) } \\
\text { same }\end{array}$ & 11 & 7 & 28 & 28 \\
\hline $\begin{array}{l}\text { minor metabolite(s) } \\
\text { different }\end{array}$ & 18 & 22 & & \\
\hline
\end{tabular}

duced by homogenate). Again here, the sensitivity of the analytical method is of importance. However, as in the case of differences between human and rat, the mere counting of similar or different metabolites may create a wrong impression about the significance of these differences. It may well be that one or two metabolites which are different between human and rat, or homogenates and microsomes, will not lead to any "real" or "really relevant" biological or toxicological consequences.

\section{Conclusions}

Several important conclusions can be made from the above described results, even before further research towards in-depth analyses and in vitro-in vivo extrapolations.

1) LC-MS -based analytical methods are efficient in the primary in vitro phase of studying organic chemicals, for both measuring the disappearance of the parent compound and the formation and tentative identification of metabolites. However, selection of chemicals for LC-MS analysis has to be made based on the type of instrument available.

2) Only 4 out of 55 preselected ECVAM/ICCVAM validation chemicals had to be changed because of analytical problems. All of them were highly halogenated compounds with poor detectability in the MS analysis.

3) Several widely spaced concentrations of the studied substance should be preferably used, even in the face of increasing costs of analyses. In this feasibility study we had to select, for cost reasons, only one concentration, $25 \mathrm{uM}$, which proved to be too low for many low-affinity compounds such as caffeine, theophylline and carbamazepine. In the in vitro phase of studies, it may not be wise to select concentrations which are in the range of anticipated in vivo concentrations.

4) A preliminary comparison of microsomes and homogenates indicated that differences were not large for most of the substances. However, it has to be recognized that microsomes as an enzyme source would not produce most phase II metabolites (e.g. paracetamol-sulphate).

5) For most compounds, microsomes are still suitable for stability and metabolism screening, but it is difficult to anticipate the extent and significance of wrong conclusions, consequent to the selection of microsomes over homogenates as an enzyme source.

6) A tentative categorical analysis indicated that differences between human and rat preparations were rather modest for most of the substances. There were a number of exceptions, e.g. amitriptyline and aflatoxin B1 regarding substrate loss. Qualitative differences in metabolite profiles were relatively common, about a third of compounds displayed a difference in major metabolite(s) and in about a half of the compounds some minor metabolites were different. However, most of these differences may not be too important. Differences were usually quantitative.

7) A tentative conclusion is that for most compounds, interspecies extrapolation between rat and human is anticipated to be relatively feasible and reliable. However, before the complete analysis of this huge data base, it is difficult to predict those compounds displaying interspecies differences of such magnitude that important pharmacological or toxicological consequences would ensue.

\section{References}

Boobis, A. R., Kremers, P., Pelkonen, O. and Pithan, K. (editors) (1999). EUR 18569 - European Symposium on the Prediction of Drug Metabolism in Man: Progress and Problems. Luxembourg: Office for Official Publications of the European Communities. 332 pp.

Coecke, S., Ahr, H., Blaauboer, B. J. et al. (2006). Metabolism: a Bottleneck in In Vitro Toxicological Test Development: The Report and Recommendations of ECVAM Workshop 54. ATLA 34, 49-84. 
Pelkonen, O. (2002). Human CYPs: in vivo and clinical aspects. Drug Metab. Rev. 34, 37-46.

Pelkonen, O., Baumann, A. and Reichel, A. (2002). Pharmacokinetic Challenges in Drug Discovery. Ernst Schering Research Foundation Workshop 37. Berlin: Springer.

Pelkonen, O., Boobis, A. R., Kremers, P. et al. (2001). Interindividual variation of P450 enzymes in vitro and its causes. In G. M. Pacifici and O. Pelkonen (eds.), Variation of Drug metabolism in Humans (269-332). London: Taylor \& Francis.

Pelkonen, O., Kaltiala, E. H., Larmi, T. K., et al. (1973). Comparison of activities of drug-metabolizing enzymes in human fetal and adult livers. Clin. Pharmacol. Ther. 14, 840-846.

Pelkonen, O., Mäenpää, J., Taavitsainen, P. et al. (1998). Inhibition and induction of human cytochrome P450 (CYP) enzymes. Xenobiotica 28, 1203-1253.

Pelkonen, O. and Raunio, H. (2005). In vitro screening of drug metabolism during drug development: Can we trust the predictions? Exp. Opin. Drug Metab. Toxicol. 1, 49-60.

Pelkonen, O., Turpeinen, M., Uusitalo, J. et al. (2005). Prediction of drug metabolism and interactions on the basis of in vitro investigations. Basic Clin. Pharmacol. Toxicol. 96, 167-175.

Salonen, J. S., Nyman, L., Boobis, A. R. et al. (2003). Comparative studies on the CYP-associated metabolism and interaction potential of selegiline between human liver-derived in vitro systems. Drug Metab. Dispos. 31, 1093-1102.
Turpeinen, M., Uusitalo, J., Jalonen, J. and Pelkonen, O. (2005). Multiple P450 substrates in a single run: rapid, comprehensive and clinically relevant in vitro interaction and metabolism assay. Eur. J. Pharm. Sci. 24, 123-132.

\section{Acknowledgements}

The studies described in this paper belong to the Project of the In-Vitro Methods Unit/ECVAM Contract No CCR.IHCP. C432889.X. Technical assistance of Ritva Tauriainen, Katri Sunnari and Birgitta Paldanius is gratefully acknowledged. The project has been partially supported by grants from the Academy of Finland and The Finnish Agency for Technology and Innovation (TEKES).

\section{Correspondence to}

Dr. Olavi Pelkonen

Department of Pharmacology and Toxicology

University of Oulu

PO Box 5000 (Aapistie 5)

FIN-90014 University of Oulu

Finland

Phone: +358-(0)8-537 5230

Fax: +358-(0)8-537 5247

e-mail: olavi.pelkonen@oulu.fi 